\title{
Droplet formation of an anisotropic liquid
}

\author{
A. Pezzutti ${ }^{1}$ and G. Araujo ${ }^{2}$ \\ 1 Instituto de Física del Sur. Universidad Nacional del Sur Av. Alem 1253. \\ 2 Universidad Nacional del Sur. Av. Alem 1253. \\ Bahía Blanca. Argentina
}

Received: March 21,2020. Revised: June 24, 2020. 2nd Revised: June 27, 2020.

Accepted: June 29, 2020. Published: June 30, 2020

\begin{abstract}
The process of formation of new morphologies by confinement in nano-droplets, created from a dewetting process, was simulated. The obtained structures showed a great similarity with the experimental results present in the literature. The developed model captures the fundamental interactions that determine the dynamics of the phase separation process of a copolymer system confined between a rigid substrate and a free surface. Furthermore, its numerical resolution is highly efficient as a result of the implementation of Eyre algorithm.
\end{abstract}

Keywords-Copolymers, Dewetting, Drop Formation, Eyre Algorithm.

\section{INTRODUCTION}

$\mathrm{T}$ he dewetting process is a phenomenon that occurs on a liquid film. Starting from a homogeneous film deposited on a substrate, the process of dewetting has as a result the rupture of the film and the droplet arrangements formation [1]. Generally, the film is prepared by spin-coating and deposited on a flat substrate. During the annealing process, the surface of the polymer is dominated by thermally excited waves.

The thermally induced $\mathrm{z}$-modulations on the liquid surface are modelled with the expression

$$
z(\vec{r}, t)=h+\delta h \exp [i \vec{q} \vec{r}]
$$

Here, $h$ is the initial film thickness.

With

$$
\delta h=\delta h_{0} \exp \left[t / \tau_{q}\right]
$$

Where $\tau_{q}$ represents the relaxation time of the $q$ modes presented by the disturbance.

Following the spinodal decomposition dynamics, the disturbances are amplified exponentially if they are less than a critical $q_{c}$ value. The fastest growing mode is:

$$
q_{m}=\sqrt{3 / 2} \frac{a}{h^{2}}
$$

With $h$ as the initial film thickness and $a$ as the molecular size. The maximum fluctuation has a characteristic time:

$$
\tau_{q_{m}}=\frac{\pi \gamma \eta}{6 a}
$$

With $\eta$ the viscosity and $\gamma$ the superficial tension. For example, in a polyethylene film [1] a typical value is $q_{m} \sim(1-$ 10) $10^{6} \mathrm{~m}^{-1}$. Instabilities cause the film to break at some points on the surface. Once the film breaks, the holes will grow proportionally to time $R \sim t$, where $R$ represents the size of the average size of the holes. The evolution and collision of the holes, transforms the film into a boundary Voronoi structure. During the subsequent evolution, the approximately toroidal boundaries decrease in a crown-shape of isolated droplets. The rupture of the boundaries is due to Rayleigh instabilities. The droplet final shape presents a contact angle determined by Young's law

$$
\cos (\theta)=\frac{\gamma_{s g}-\gamma_{s l}}{\gamma_{l g}}
$$

Where $\gamma_{s g}, \gamma_{s l}$ and $\gamma_{l g}$ represent superficial tensions between solid-gas, solid-liquid and liquid-gas interfaces, respectively. Typically for a PS film, the contact angle is between 8 and 12 degrees [1].

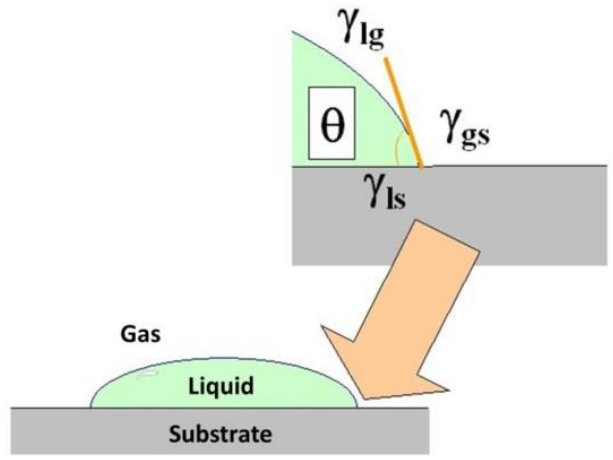

Fig. 1. Contact angle. The contact $\theta$ angle of the liquid droplet formed after the dewetting process is determined by Young's equation. In the graph, $\gamma_{l g}$ represents the liquid-gas surface tension, $\gamma_{l s}$ represents the liquid-solid surface tension, and $\gamma_{s g}$ represents the solid-gas surface tension.

The dewetting process can be seen in figure 2. The images correspond to a dewetting process of a PS film. The figure is extracted from the work done by Reiter [1].

When the liquid used is homogeneous, the shape of the droplet is determined by the surface tensions according to Young's equation. However, if the composition of the liquid is 
anisotropic, the shape of the droplet changes. The competition between the surface tensions and the internal structure determines the ultimate droplet shape. For example, by anisotropy effects, droplet formation with pyramidal structure has been observed in lamella phase copolymer systems [2].

Confining a copolymer alters its structure in volume. By using a dewetting process, nano-rings, obtained by confining a mixture of copolymers with a hexagonal structure in nanodroplets [3], have been manufactured.

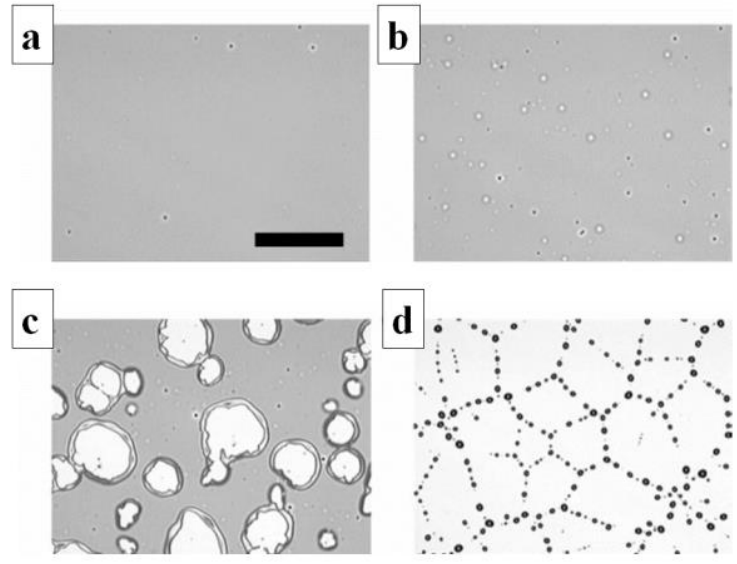

Fig. 2. Optical microscopy image. Dewetting process of a PS film, supported on a silicon substrate.

a- Thermal instabilities propagate on the film surface causing it to break into small holes. b- The size of the holes increases with time. c-

The collision of the holes causes boundaries formation. $\mathbf{d}$ - The

advanced stage of dewetting ends with the destabilization of the boundaries forming small drops. The figure has been extracted from the work realized by Reiter [1].

\section{COPOLYMER NANO-DROP MODEL}

\section{A. Copolymer-Solvent System}

We can express the free energy of a ternary copolymersolvent system as a function of the order parameters $\eta$ and $\phi$, where $\phi$ represents the composition of the copolymer mixture and $\eta$ represents the solvent [4,5].

Energy is composed of a short-range term $F_{S}[\phi, \eta]$ and a longrange term $F_{l}[\phi]$. The short-range term is:

$$
\begin{gathered}
F_{S}[\phi, \eta]=\int d r\left[f_{\eta}(\eta)+f_{\phi}(\phi)+W(\phi, \eta)+\right. \\
\left.\frac{D_{1}}{2}|\nabla \eta|^{2}+\frac{D_{2}}{2}|\nabla \phi|^{2}\right]
\end{gathered}
$$

Where $D_{1}$ and $D_{2}$ are phenomenological parameters related to interface energy. The expressions $f_{\eta}(\eta), f_{\phi}(\phi)$ refer to double-well potentials, specifically:

$$
\begin{gathered}
f_{\eta}(\eta)=-\frac{1}{2} c_{1} \eta^{2}+\frac{u_{1}}{4} \eta^{4} \\
f_{\phi}(\phi)=-\frac{1}{2} c_{2} \phi^{2}+\frac{u_{2}}{4} \eta \phi^{4}
\end{gathered}
$$

Where $c_{i}$ and $u_{i}$ are phenomenological constants.

The expression $W(\phi, \eta)$ indicates the potential of interaction between both order parameters. It is given by:

$$
W(\phi, \eta)=b_{1} \phi \eta-\frac{b_{2}}{2} \eta \phi^{2}+\frac{b_{4}}{2} \eta^{2} \phi^{2}-\frac{1}{2} b_{3} \eta^{2} \phi
$$

The long-range term is:

$$
F_{l}[\phi]=\int d \boldsymbol{r} \int d \dot{\boldsymbol{r}} G(\boldsymbol{r}, \dot{\boldsymbol{r}})(\phi(\boldsymbol{r})-\bar{\phi})(\phi(\dot{\boldsymbol{r}})-\bar{\phi})
$$

The expression above is related to the $\phi$ parameter that represents the copolymer.

The interaction potential $b_{i}$ parameters characterize the interactions between copolymer blocks and the solvent.

Time evolution results in a set of coupled differential equations for conserved order parameters:

$$
\begin{gathered}
\frac{\partial \eta}{\partial t}=\nabla^{2}\left(\frac{\delta F}{\delta \eta}\right) \\
\frac{\partial \phi}{\partial t}=\nabla^{2}\left(\frac{\delta F}{\delta \phi}\right)
\end{gathered}
$$

\section{B. Simulation of Copolymer-Solvent System}

The Eyre algorithm previously developed [6, 13] for a copolymer system can be adapted to solve the set of CahnHilliard equations that model the time evolution of the copolymer and the solvent equations (11) and (12).

The algorithm expression is:

$$
\begin{array}{r}
\phi_{t+\Delta t}-\Delta t \nabla^{2}\left(-\left(1-a_{1}\right) c_{2} \phi_{t+\Delta t}-D_{2} \nabla^{2} \phi_{t+\Delta t}\right)+ \\
\Delta t \alpha \phi_{t+\Delta t}=\phi_{t}+\Delta t \nabla^{2}\left(-a_{1} c_{2} \phi_{t}+u_{2} \phi_{t}^{3}+b_{1} \eta-\right. \\
\left.b_{2} \eta \phi-\frac{b_{3}}{2} \eta^{2}+b_{4} \eta^{2} \phi\right)-\alpha \Delta t \bar{\phi}
\end{array}
$$

$$
\begin{array}{r}
\eta_{t+\Delta t}-\Delta t \nabla^{2}\left(-\left(1-a_{2}\right) c_{1} \eta_{t+\Delta t}-D_{1} \nabla^{2} \eta_{t+\Delta t}\right)= \\
\phi \eta_{t}+\Delta t \nabla^{2}\left(-a_{2} c_{1} \eta_{t}+u_{1} \eta_{t}^{3}+b_{1} \phi-\frac{b_{2}}{2} \phi^{2}-b_{3} \phi \eta+\right. \\
\left.b_{4} \eta \phi^{2}\right)-\beta \Delta t(\phi-\bar{\phi})
\end{array}
$$

With the following stability conditions

$$
a_{1} \geq \frac{1}{c_{2}}\left[\frac{c_{2}}{2}+\frac{3}{2} b_{2}+3 u_{2}\right]
$$




$$
a_{2} \geq \frac{1}{c_{1}}\left[\frac{c_{1}}{2}+\frac{3}{2} b_{3}+3 u_{1}\right]
$$

To illustrate this point, we simulate the time evolution of a two-dimensional solvent copolymer system. Figure (3) and (4) illustrate the formation of solvent immersed copolymer domains.

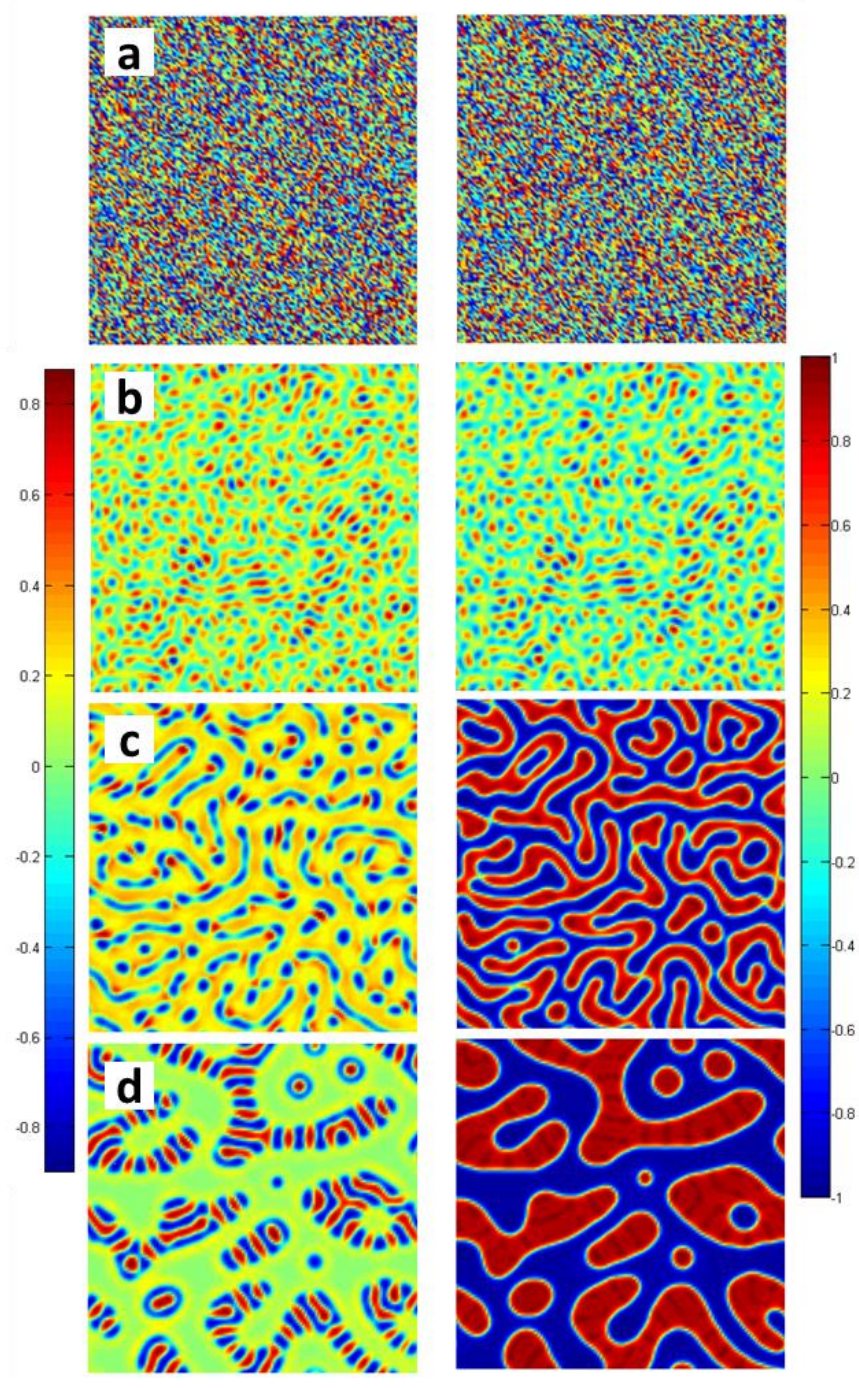

Fig. 3. Time evolution of the copolymer-solvent system. The figures show the order parameter of copolymer $\phi$ on the left and the order parameter of solvent $\eta$ on the rigth. Simulation data: system with a $512 \times 512$ grid with $\Delta \mathrm{x}=0.5, \Delta \mathrm{t}=1.5$ and periodic boundary conditions for both order parameters. $\mathrm{c}_{2}=0.1, \mathrm{u}_{2}=1, \mathrm{D}_{2}=1, \alpha=1, \phi=0$ was used for the copolymer. For this set of parameters, the structure corresponds to a lamellae structure. $\mathrm{c}_{1}=0.3, \mathrm{u}_{1}=1, \mathrm{D}_{1}=0.5$ was used for the solvent. The interaction parameters were $b_{1}=0.07, b_{2}=0.3, b_{3}=0, b_{4}=0.1$. Times:

(a) $\mathrm{t}=0$, (b) $\mathrm{t}=10$, (c) $\mathrm{t}=1000$, (d) $\mathrm{t}=10000$. The figure has been extracted from [6].

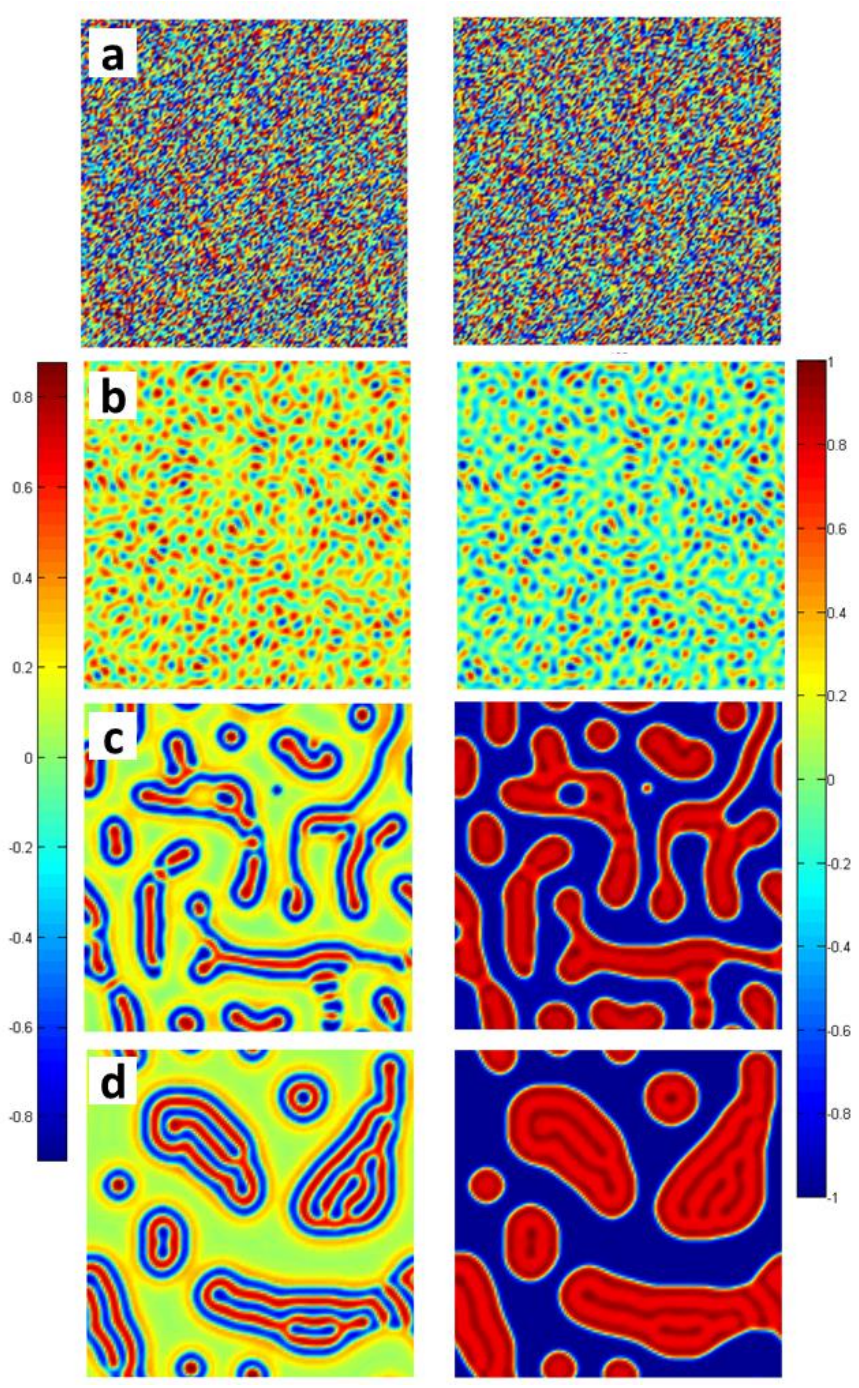

Fig. 4. Time evolution of the copolymer-solvent system. The figures show the order parameter of copolymer $\phi$ on the left and the order parameter of solvent $\eta$ on the rigth. Simulation data: system with a $512 \times 512$ grid with $\Delta x=0.5, \Delta t=1.5$ and periodic edge conditions for both order parameters. $c_{2}=0.1, u_{2}=1, D_{2}=1, \alpha=1, \phi=0$

was used for the copolymer. For this set of parameters, the structure corresponds to a lamellae structure. $c_{1}=0.3, u_{1}=1, D_{1}=0.5$ was used for the solvent. The interaction parameters were $b_{1}=0.03, b_{2}=0.3, b_{3}=0, b_{4}=0.1$. Times:

(a) $t=0$, (b) $t=10$, (c) $t=1000$, (d) $t=10000$.

Figure (3) illustrates the time evolution of the copolymersolvent system phase separation. The $b_{1}$ parameter determines the preference or affinity of the solvent for one of the copolymer blocks. For the value $b_{1}=0.07$, there is a strong affinity with one of the blocks, which results in the formation of copolymer micelles. It should be noted that in most parts of macrodomains, the lamellar domains tend to be parallel to the macrophase interfaces and the domains is surrounded by a thin layer of one block. The decrease in the $b_{1}$ value to a $b_{1}=$ 0.03 value is illustrated in Figure (4). A lower $b_{1}$ value results 
in a similar affinity between the copolymer blocks and the solvent. As seen in Figure (4) the interface of the copolymer domains is uniformly composed by both blocks. For this value the stripe domains emerge perpendiculary to the macrophase interfaces. Thus there is a clear morphological transition by changing the interaction parameter $b_{1}$. Parameter $b_{1}$ allows for the interaction between the solvent and the copolymer blocks to be modeled. The morphological change occurs at about $b_{1}=0.04$. This behavior coincides qualitatively with the experimental results.

\section{Simulation of Copolymer Nano-Droplets on Rigid} Substrate.

To simulate the temporal evolution of a copolymer droplet deposited on a rigid substrate, the air copolymer system was modeled as a ternary system (solvent copolymer), where the air was treated as a bad solvent. The interaction with the substrate was simulated through a potential of the form:

$$
P(x, y, z)=P_{0} \exp \left[-z / z_{0} \phi\right]
$$

Where the $P_{0}$ sign determines the preference towards one of the blocks of the copolymer. The $z_{0}$ term determines the extent of the interaction potential. The $\mathrm{z}$ coordenate refers to the normal direction to the substrate. Figure (5) illustrates the represented system.

\section{Air (bad solvent)}

\section{Copolymer}

\section{Substrate}

Fig. 5. Schematic representation of the simulated system. A droplet of copolymer on a rigid substrate.

The system was simulated with a $128 \times 128 \times 64(\mathrm{x}, \mathrm{y}, \mathrm{z})$ grid with $\Delta x=\Delta y=\Delta z=0.3$ and $\Delta t=2$. Periodic boundary conditions in the $\mathrm{x}$ and $\mathrm{y}$ direction and no flow in the $\mathrm{z}$ direction were used. Eyre algorithm developed in section $\mathrm{B}$ was used for time evolution, and spatial derivatives were resolved using a spectral method [6].

\section{RESULTS AND DISCUSSION}

The interaction between the copolymer and the substrate was simulated by the external potential presented in equation 17 . To study the effects of the substrate on the morphologies obtained, different values for the $P_{0}$ interaction were used. To the $\boldsymbol{z}_{\mathbf{0}}$ parameter was assigned a value of 0.1 , which limits the interaction of the potential to an extremely small distance near the wall, much less than the radius of gyration of the molecules. The interaction between the air and the copolymer was modeled by considering air as a bad solvent, i.e. with a low affinity between the solvent and the copolymer. For this purpose, the values of the interaction potential were chosen appropriately. The interaction parameters $b_{2}=0.5, b_{3}=$ 0 and $b_{4}=1$ were chosen to obtain low affinity between the solvent (air) and the copolymer.

The effect of confinement for lamellae bulk structures, cylinders and BCC structures was studied.

$c_{1}=0.1, u_{2}=1, D_{2}=1$ y $\alpha=1, \bar{\phi}=0$ was used for the lamellae system.

$c_{1}=0.1, u_{2}=1, D_{2}=1$ y $\alpha=1, \bar{\phi}=0.05$ was used for the cylinder system

$c_{1}=0.1, u_{2}=1, D_{2}=1$ y $\alpha=1, \bar{\phi}=0.1$ was used for the $\mathrm{BCC}$ system.

The initial condition is illustrated in Figure (6), and represents the conformation of a disorderly phase copolymer confined to a nano-droplet deposited on a substrate.

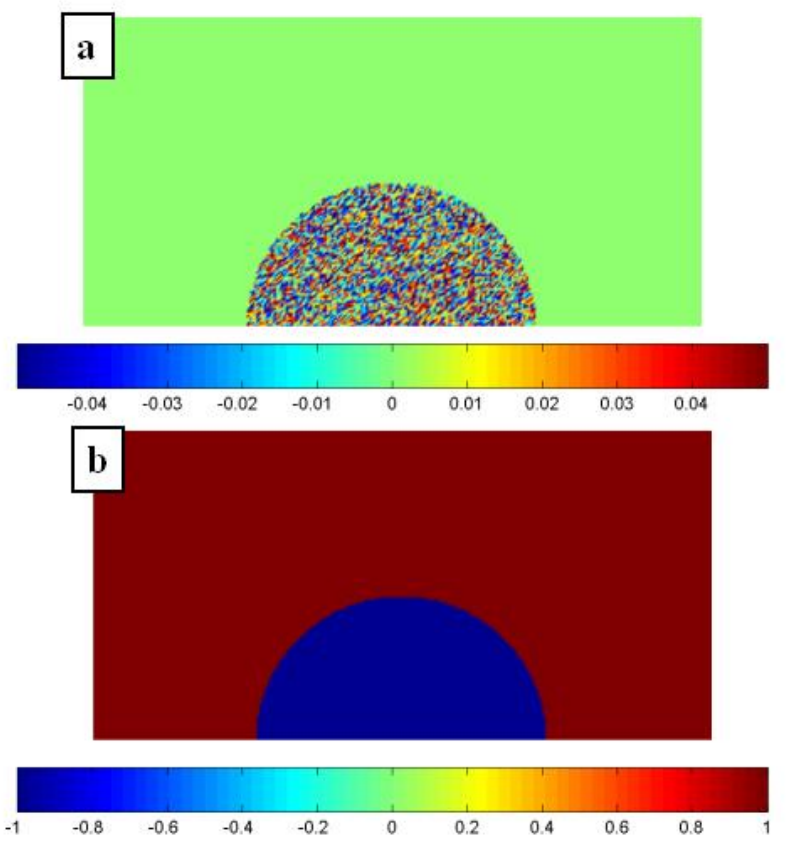

Fig.6. Initial conditions of order parameters $\phi$ and $\eta$ (a) and (b) respectively. The initial condition represents a droplet of disorderly phase copolymer supported on a substrate.

The lamellar phase was simulated for different values of $b_{1}$ and $P_{0}$. The above parameters represent the affinity of one of the copolymer blocks with the air and the substrate. The relationship between the two generates different morphologies of the copolymer droplets. The time evolution of a nano-drop with lamellar internal structure is illustrated in the Figure (7). Figure (8) show the evolution of shape. The interaction 
between the surface tension of the copolymer and solvent system and the internal structure, cause a transformation of the drop shape from an initial semi-sphere shape to a pyramidal shape.
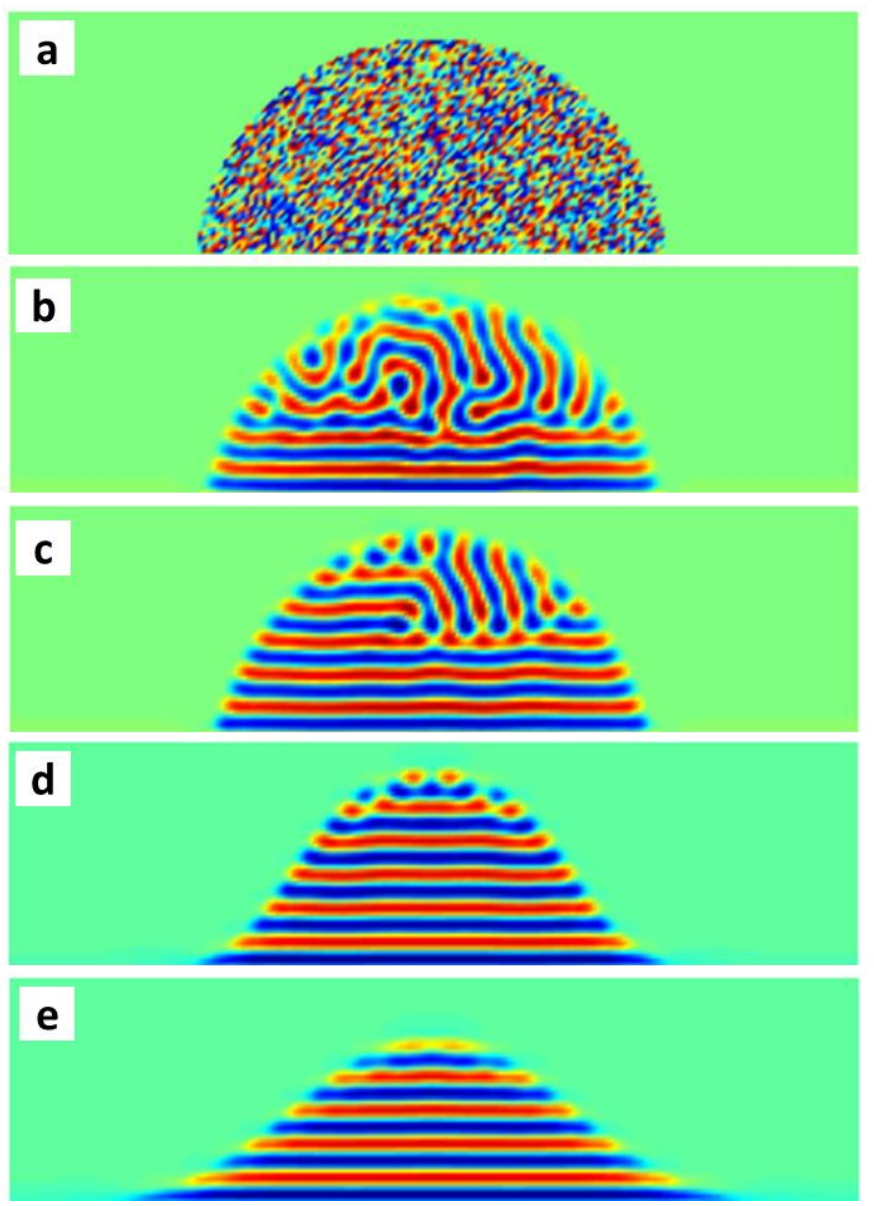

Fig. 7.Time evolution of copolymer nano-drops on a rigid substrate. The composition of the copolymer is shown in the $x, z$ plane.

Simulation data: system with a $512 \times 512 \times 256$ grid with $\Delta x=0.3, \Delta t=$ 1.5 and periodic boundary conditions in the $x$ and $y$ direction and no flow in the $z$ direction. $c_{2}=0.1, u_{2}=1, D_{2}=1, \alpha=1, \phi=0$ was used for the copolymer. For this set of parameters, the structure corresponds to a lamellae structure. $c_{1}=0.3, u_{1}=1, D_{1}=0.5$ was used for the solvent. The interaction parameters were $b_{1}=0.03, b_{2}=0.3, b_{3}$ $=0, b_{4}=0.1$. Times: (a) $t=0$, (b) $t=1000$, (c) $t=10000$, (d) $t=$ 100000 .

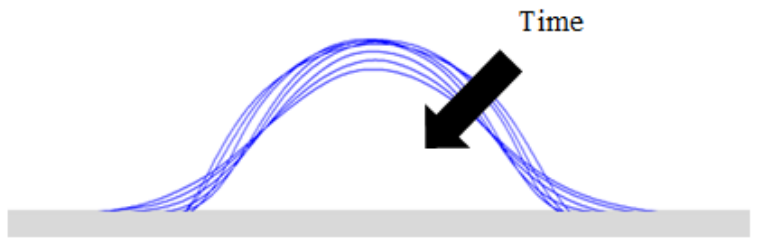

Fig. 8.Time evolution of copolymer nano-drops on a rigid substrate. The interaction between the surface tension of the copolymer and solvent system and the internal structure, cause a transformation of the drop shape from an initial semi-sphere shape to a pyramidal shape.
Figure (9) illustrates the different structures developed by the copolymer confined in a nano-droplet. In all cases, the droplet has a pyramidal type shape, induced by the lamellar structure of the copolymer. A parallel orientation to the substrate is observed in all cases. Experimental work in the literature shows a similar formation of structures within the nanodroplets. Figure (10), specially, shows the image of a nanodroplet obtained by the dewetting process of a diblock polystyrene-polymethyl methacrylate (PS-PMMA) copolymer in the lamellae phase. Details can be found in the work of Croll et al.[2,7,12].
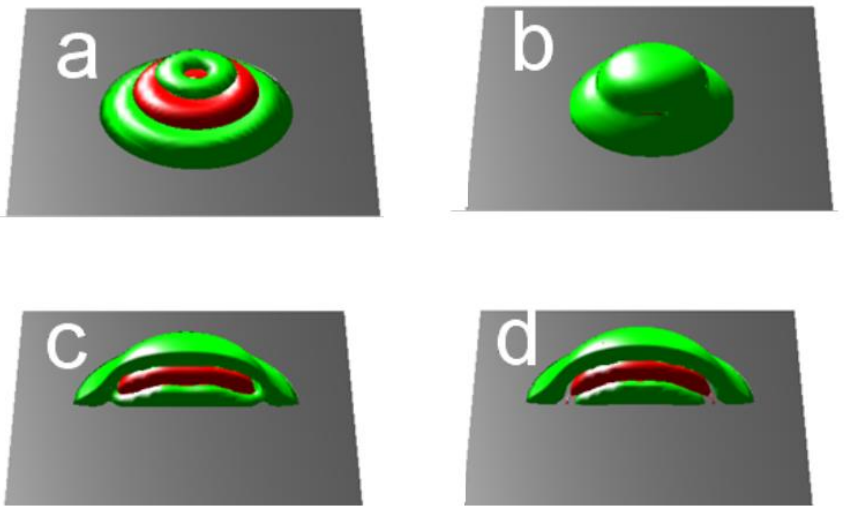

Fig. 9. Nano-droplet structures obtained for a bulk phase copolymer of lamellae. The images reflect the interaction of the substrate and the free surface, which determine the final shape of the nano-droplet. The values of the interaction are a- $P_{0}=0.01, b_{1}=0.03$, b- $P_{0}=$ $0.01, b_{1}=0.03$ c- $P_{0}=0.01, b_{1}=0.05$ d- $P_{0}=0.02, b_{1}=0.02$.

Figures $\mathrm{c}$ and $\mathrm{d}$ show a cut to observe inside the droplet.

Note the excellent alignment between the results obtained from the simulation (Figure 9 panel a), and the experimental data reported in the literature, shown in Figure (10). The BCC phase was simulated for different $b_{1}$ and $P_{0}=0.02$ values. In Figure (11), the different obtained morphologies are illustrated.

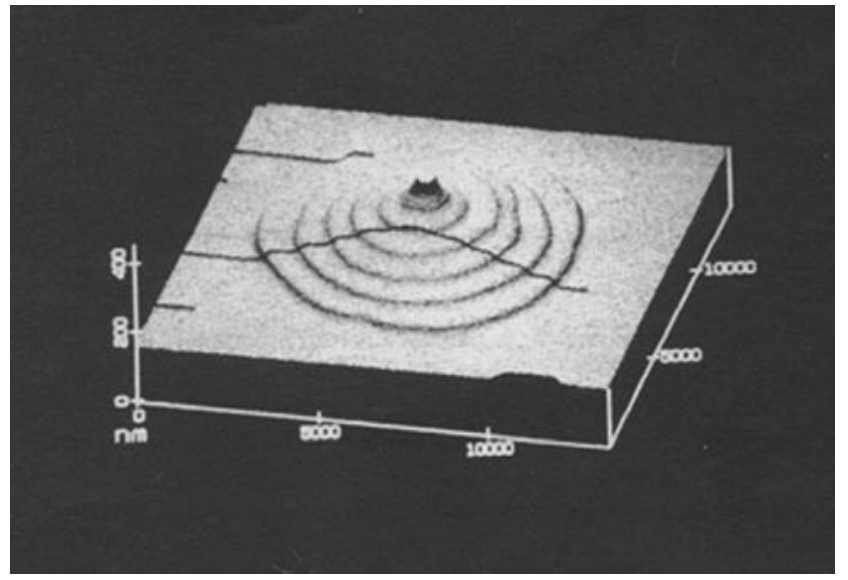

Fig. 10. AFM image. Nano-droplet of copolymer in lamellae phase obtained by the dewetting process. The image shows the stepwise shape of the droplet induced by the competition between the surface tension on the liquid surface and the lamellae structure forming the copolymer droplet. The image was extracted from the work of Croll et al. [2]. 
In this case, the morphologies correspond to a fixed value of interaction with the substrate and different values of interaction with the air or the solvent. When the air interaction is smooth, the droplets morphology presents a great similarity with those obtained in spherical confinement for copolymers $[7,9,11]$ in BCC phase (Figure 11, panels $a$ and $b$ ). With strong air interaction, the BCC structure is strongly altered, forming a concentric lamellar structure (Figure 11 panel c).
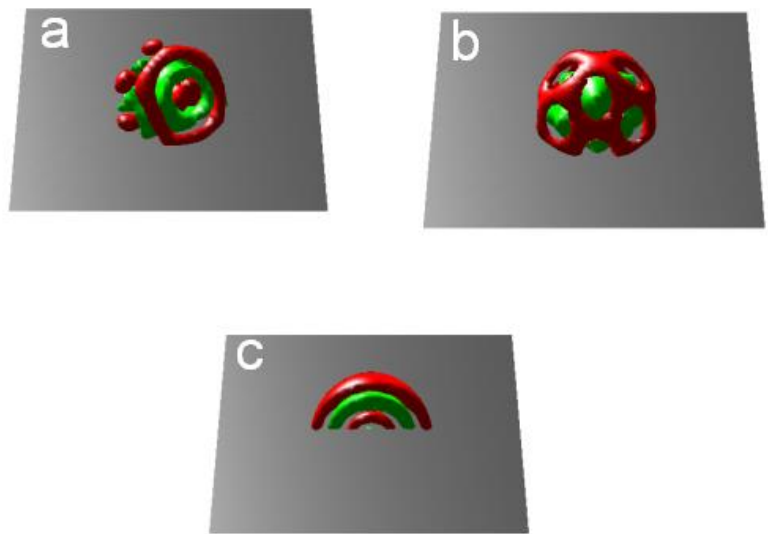

Fig. 11. Nano-droplet structures obtained for a bulk phase copolymer of BCC spheres. The images reflect the interaction of the substrate and the free surface, which determine the final shape of the nanodroplet. The interaction values are a- $P_{0}=0.02, b_{1}=0.01$ b- $P_{0}=$ $0.02, b_{1}=0.01$ c- $P_{0}=0.012 b_{1}=0.06$. Figure $\mathrm{c}$ is shown in cut to observe the inside structure.

The hexagonal phase was simulated for different $b_{1}$ and $P_{0}=$ 0.02 values. The different structures developed by the copolymer confined in a nano-droplet are illustrated in Figure (12) where the images correspond to increasing $b_{1}$ values. The strong confinement induced by the substrate and the air produces a marked alteration of the hexagonal phase. Due to bending effects, the cylinders are transformed into rings parallel to the substrate. Experimental works present in literature show a similar formation of nano-rings obtained in droplets generated by dewetting $[3,10]$. Particularly, the image of a nano-droplet obtained by the PS-PS copolymer dewetting process in hexagonal phase of cylinders is presented in Figure (13). Details are available in the work of Shaw et al. [3]. Note the excellent alignment between the experimental results present in the literature and the simulations performed.
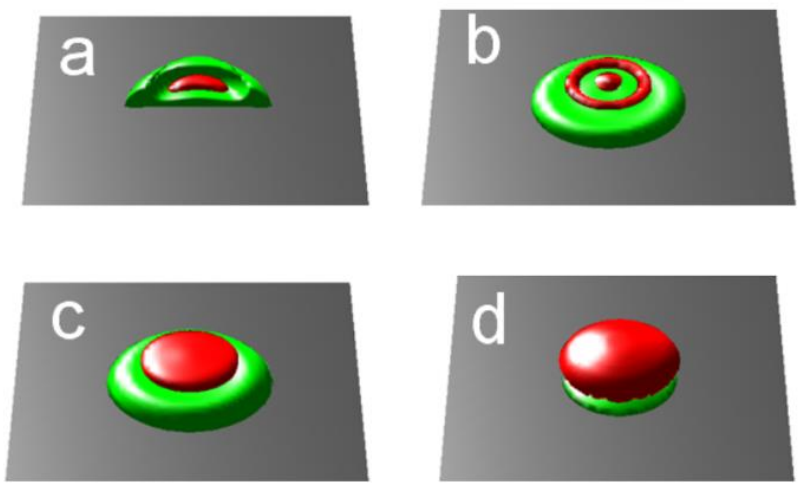

Fig. 12. Non-droplet structures obtained for a bulk phase copolymer of hexagonal arrangements. The images reflect the interaction of the substrate and the free surface, which determine the nano-droplet final shape. The interaction values are a- $P_{0}=0.02, b_{1}=0.01$ b- $P_{0}=$ $0.02, b_{1}=0.02$ c- $P_{0}=0.02 b_{1}=0.03$ d- $P_{0}=0.02, b_{1}=0.04$.
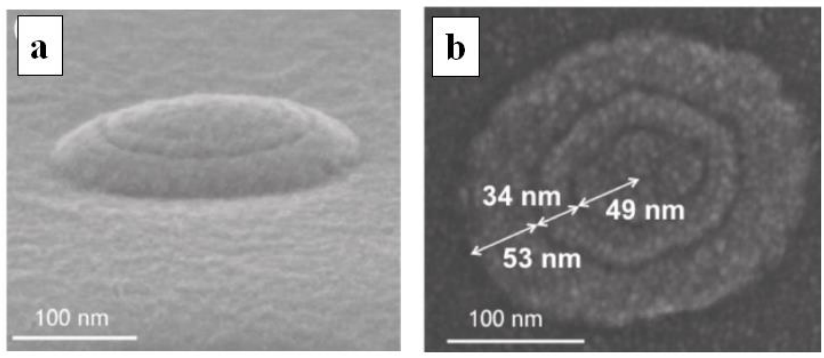

Fig- 13. TEM image. Nano-droplet of copolymer in phase of cylinders in hexagonal arrangements obtained by the dewetting process. The strong confinement transforms the copolymer bulk structure. The process allows to produce nano-rings. Experimental details are available in the work done by Shaw et al [3].

\section{CONCLUSION}

Bulk morphologies of diblock copolymers have been extensively studied in the literature both experimentally and theoretically. Depending on the composition of the blocks it is comprised of, the bulk structures correspond to lamellae, gyroids, cylinder hexagonal arrangements and $\mathrm{BCC}$ sphere structures. Recent research has shown that confinement is a powerful tool to break the symmetry of a structure, and obtain new shapes, different from those of bulk. The process of formation of new morphologies by confinement in nanodroplets, created from a dewetting process, was simulated. The obtained structures showed a great similarity with the experimental results present in the literature. The developed model captures the fundamental interactions that determine the dynamics of the phase separation process of a copolymer system confined between a rigid substrate and a free surface. Furthermore, its numerical resolution is highly efficient as a result of the implementation of Eyre algorithm. In future work, we will apply this method to study the processes of formation of nano-drops from the dewetting process, as well as the formation of new structures generated by confinement. 


\section{ACKNOWLEDGMENT}

This work was supportedby Universidad Nacional del Sur, and the National Research Council of Argentina (CONICET).

\section{References}

[1] Günter Reiter. Dewetting of thin polymer films. Phys. Rev. Lett., 68:75-78, Jan 1992.

[2] Andrew B. Croll, Michael V. Massa, Mark W. Matsen, and Kari Dalnoki-Veress. Droplet shape of an anisotropic liquid. Phys. Rev.Lett., 97:204502, Nov 2006.

[3] Matthew T. Shaw Vincent Reboud Marc Zelsmann Justin D.Holmes Clivia M. Sotomayor Torres Richard A. Farrell, Nikolaos Kehagias and Michael A. Morris. Surface-directed dewetting of a block copolymer for fabricating highly uniform nanostructured microdroplets and concentric nanorings. ACS Nan, 5(2):1073,2011.

[4] A Ito, Domain patterns in copolymer-homopolymer mixtures, Physical Review E 58, 98 (1998).

[5] T Otha and A Ito, Dynamics of phase separation in copolymer-homopolymer mixtures, Physical Review E 52, 5250 (1995).

[6] A. D. Pezzutti and H. Hernández. Unconditionally Stable Algorithm for Copolymer and Copolymer-Solvent Systems, Papers in Physics (under review).

[7] Peng Chen and Haojun Liang. Origin of microstructures from confined asymmetric diblock copolymers. Macromolecules, 40:7329, 2007.

[8] Brassat, K., and Lindner, J. K. Nanoscale Block Copolymer Self-Assembly and Microscale Polymer Film Dewetting: Progress in Understanding the Role of Interfacial Energies in the Formation of Hierarchical Nanostructures. Advanced Materials Interfaces, 7(5), 1901565 (2020).

[9] Wang, J., Li, Y., Zheng, L., Wu, L., Wang, H., \& Zhang, $\mathrm{X}$. Self-assembly behaviors of graft copolymer structured fluid droplets on flat solid surfaces. Chemical Physics Letters, 721, 43-48 (2019).

[10] Majewski, P. W., \& Yager, K. G. Rapid ordering of block copolymer thin films. Journal of Physics: Condensed Matter, 28, 403002 (2016).

[11] Doerk, G. S., \& Yager, K. G. Beyond native block copolymer morphologies. Molecular Systems Design \& Engineering, 2, 518-538 (2017).

[12] Onses, M. S., Song, C., Williamson, L., Sutanto, E., Ferreira, P. M., Alleyne, A. G. \& Rogers, J. A. Hierarchical patterns of three-dimensional block-copolymer films formed by electrohydrodynamic jet printing and self-assembly. Nature nanotechnology, 8, 667-675 (2013).

[13] Aldo Pezzutti and Gastón Araujo, Crumpling and Buckling in Membranes with Hexagonal Phase,

WSEAS Transactions on Applied and Theoretical Mechanics, 15, 98-107 (2020).
[14] Alexander Blokhin, Ekaterina Kruglova, Boris Semisalov, Numerical Analysis of the Non-Isothermal Flow of Polymeric Liquid between Two Coaxial Cylinders, WSEAS Transactions on Fluid Mechanics, pp. 26-36, Volume 13, 2018 ,

\section{Creative Commons Attribution License 4.0 (Attribution 4.0 International, CC BY 4.0)}

This article is published under the terms of the Creative Commons Attribution License 4.0 https://creativecommons.org/licenses/by/4.0/deed.en US 